\title{
Calculating Reproductive Performance in Beef Operations: The University of Florida Beef Herds' 2019 Breeding Season ${ }^{1}$
}

\author{
Mario Binelli, Angela M. Gonella-Diaza, Thiago Martins, Cecilia C. Rocha, Felipe A. C. C. \\ Silva, Federico Tarnonsky, Sergio Roskopf, Owen Rae, Danny Driver, Nicolas DiLorenzo, Jose \\ Dubeux, and David Thomas ${ }^{2}$
}

\section{Introduction}

Cow-calf operations are predominant in Florida. For most producers, the bottom line is how many pounds of weaned calves they will be able to market in a given year. While many factors impact that endpoint, reproductive performance comes first: no calf, no revenue. Fewer calves, less revenue. Lighter calves, less revenue. It is that simple. However, when it comes to reproductive performance, there is much confusion and even misinformation among producers. Much of the confusion stems from two specific points: how to calculate reproductive performance and how to evaluate performance data. The first objective of this publication is to review the basic calculations of reproductive performance. After producers calculate the reproductive performance of the herd, they need a reference point to compare their results. The second objective of this article is to report the reproductive performance of two beef herds of the University of Florida, located in north (UF/IFAS NFREC in Marianna) and central Florida (Beef Units in Gainesville). This report will be updated annually to provide a moving benchmarking target for the industry in Florida. A companion EDIS publication, Calculating Calf Performance in Beef Operations: The University of Florida Beef Herds 2019-2020 Calving Season, addresses a second component of reproductive performance affecting producers' bottom line, which is calf performance. This report is intended to be used by county faculty educating producers on the subject of reproductive performance and by producers who may need a reference point to benchmark the performance of the operation.

\section{Reproductive Performance Calculations}

Regarding the calculations of reproductive performance, the simplest measure is Pregnancy Rate. In systems with a defined breeding season, Pregnancy Rate is:

Pregnancy Rate $(\%)=$ Number of females pregnant $/$ Number of females that entered the breeding season (x100)

For operations without a defined breeding season, use a limited time frame for calculation (e.g., a 12-month period). Pregnancy Rates are usually calculated after a

1. This document is AN365, one of a series of the Department of Animal Sciences, UF/IFAS Extension. Original publication date May 2021. Visit the EDIS website at https://edis.ifas.ufl.edu for the currently supported version of this publication.

2. Mario Binelli, PhD, assistant professor, Department of Animal Sciences; Angela M. Gonella-Diaza, DVM, PhD, assistant professor, UF/IFAS North Florida Research and Education Center; Thiago Martins, DVM, PhD, postdoctoral fellow, Department of Animal Sciences; Cecilia C. Rocha, DVM, Department of Animal Sciences; Felipe A. C. C. Silva, DVM, Department of Animal Sciences; Federico Tarnonsky, DVM, UF/IFAS NFREC; Sergio Roskopf, DVM, UF/ IFAS NFREC; Owen Rae, DVM, professor, Department of Large Animal Clinical Sciences, UF College of Veterinary Medicine; Danny Driver, MSc, general manager, Department of Animal Sciences; Nicolas DiLorenzo, PhD, associate professor, UF/IFAS NFREC; Jose Dubeux, PhD, associate professor, UF/IFAS NFREC; and David Thomas, general manager, UF/IFAS NFREC; UF/IFAS Extension, Gainesville, FL 32611.

The Institute of Food and Agricultural Sciences (IFAS) is an Equal Opportunity Institution authorized to provide research, educational information and other services

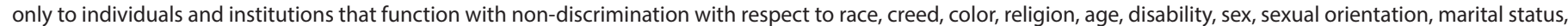

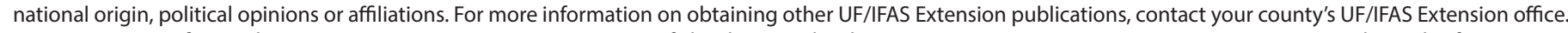
U.S. Department of Agriculture, UF/IFAS Extension Service, University of Florida, IFAS, Florida A \& M University Cooperative Extension Program, and Boards of County Commissioners Cooperating. Nick T. Place, dean for UF/IFAS Extension. 
pregnancy diagnostic that is conducted at least 30 days after the end of the breeding season. For herds that utilize artificial insemination (AI), it is usual to calculate the Pregnancy Rate to that insemination. In that situation, a pregnancy test is usually conducted 30 days after the AI. Other common measures of reproductive performance are:

Calving Rate $(\%)=$ (Number of calves born / Number of females that entered the breeding season) $\times 100$

Weaning Rate $(\%)=($ Number of calves weaned / Number of females that entered the breeding season) $\times 100$

Pregnancy Maintenance $(\%)=($ Number of calves born $/$ Number of females diagnosed as pregnant) $\times 100$

Pregnancy Loss $(\%)=100$ - Pregnancy maintenance (\%)

Calf Survival $(\%)=($ Number of calves weaned $/$ Number of calves born) $\times 100$

Calf Loss (\%) $=100-$ Calf Survival (\%)

\section{Reproductive Performance of UF Beef Herds}

Producers always ask what numbers they should aim for. There is no definitive answer, because different performances are expected from different production systems. Producers may find it useful to utilize the numbers from this report as a reference point to gauge the reproductive performance of their operations. To that end, it is always useful to look at real numbers obtained from reliable sources. Here, we present a yearly report of the reproductive performance of the University of Florida beef herds. Each University herd operates according to its respective regional characteristics and specific management (Table 1 and Figure 1). Figure 2 presents the synchronization protocol, reproductive performance, pregnancy maintenance, and calf survival data for both the Gainesville (UF/ IFAS Beef Unit) and the Marianna (UF/IFAS North Florida Research and Education Center) herds for the 2019 breeding season/2019-2020 calving season.

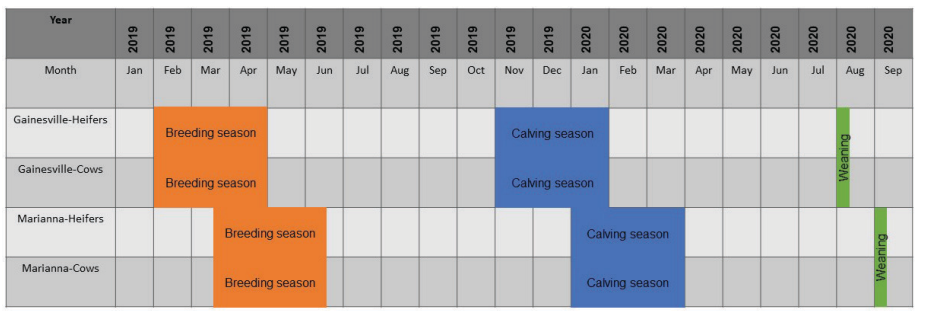

Figure 1. Calendar distribution of the breeding season (orange), calving season (blue), and weaning (green bar) on the UF/IFAS beef herds. In both herds, the breeding season starts when females are artificially inseminated, following a synchronization protocol (Figure 2). Bulls are introduced a few days after Al (Table 1) and removed at the end of the breeding season. The calving season is consistent with the average gestation length in cattle (283 days). Because all females are inseminated on day 1 of the breeding season, Al calves are born first and calves from natural service follow.

Credits: Binelli's Lab, UF/IFAS
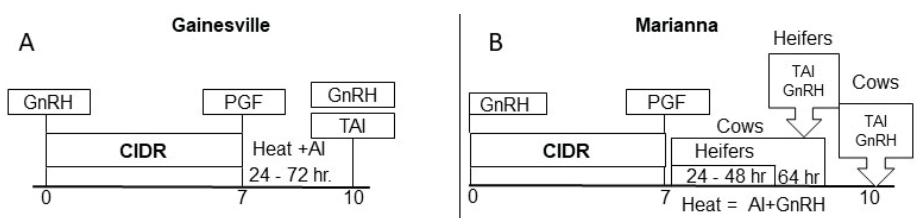

C
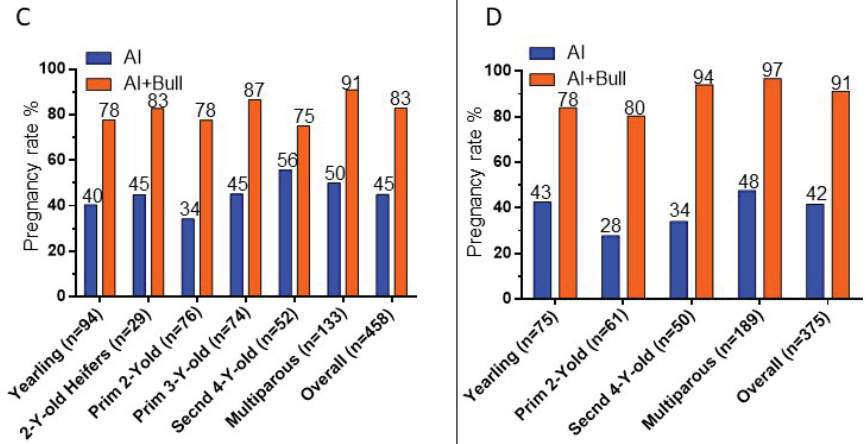

E Calves/Pregnant
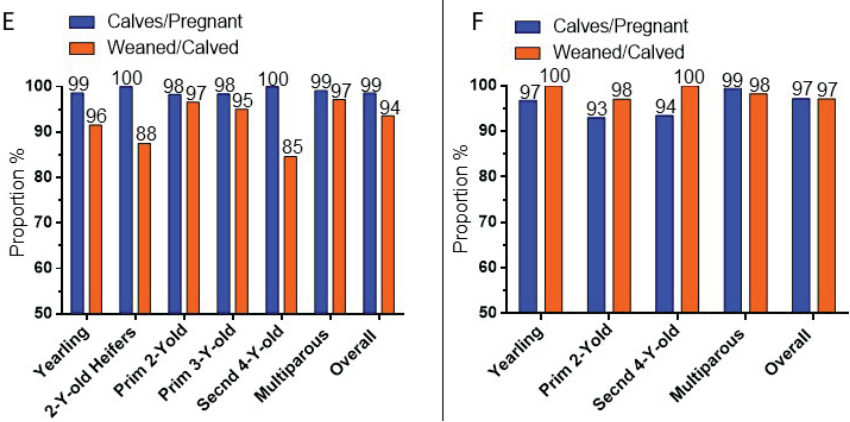

Figure 2. Synchronization protocol, Pregnancy Rate, Pregnancy Maintenance (number of calves born alive divided by number of females pregnant), and Calf Survival (number of calves weaned divided by number of calves born alive) for the Gainesville (panels $A$, $C$, and E) and the Marianna herds (panels B, D, and F). Credits: Binelli's Lab, UF/IFAS

\section{The Gainesville Herd (UF/IFAS Beef Units)}

There are two main beef herds in Gainesville: the purebred Brahman herd and the Multibreed herd. Only data for the Multibreed herd are presented here (for approximately 450 females). The Multibreed herd is composed of crosses of Angus and Brahman in the following proportions: 0\%-20\% Brahman, 20\%-40\% Brahman, 40\%-60\% Brahman, 60\%-80\% Brahman, and 80\%-100\% Brahman. Every female entering the breeding season is synchronized using the Select Synch + CIDR \& TAI protocol (Figure 2A). Specifically, after removal of the CIDR, females are observed for heat at least twice daily for 72 hours and inseminated using the AM/PM rule. Females not observed in heat receive timed artificial insemination (TAI) 80 hours after CIDR removal. Semen is mostly from University of Florida bulls. Fifteen days after AI, females are placed in natural service breeding groups and bred to clean-up bulls of the same approximate breed composition as the AI sires used on each cow group, until the end of the breeding season. Pregnancies are diagnosed twice, approximately 
45 days and 150 days after AI. Calves are identified and processed within 24 hours of birth. All calves are weaned together at approximately 7-8 months of age.

\section{The Marianna Herd (UF/IFAS NFREC)}

The Marianna herd is composed of approximately $350 \mathrm{com}$ mercial Angus, Brangus, and Braford females. Every female entering the breeding season is synchronized using the Select Synch + CIDR \& TAI protocol (Figure 2B). Specifically, after removal of the CIDR, females are observed for heat at least twice daily for 60 hours and inseminated using the $\mathrm{AM} / \mathrm{PM}$ rule. Females not observed in heat receive timed artificial insemination 64 hours (heifers) or 72 hours (cows) after CIDR removal. Semen is mostly from commercial bulls. Four to eight days after AI, females are placed in natural service breeding groups and bred to clean-up bulls of the same breed as the AI sires used on each cow group, until the end of the breeding season. Pregnancies are diagnosed twice, approximately 30 days and 120 days after AI. Calves are identified and processed within 24 hours of birth. All calves are weaned together at approximately 7-8 months of age. The UF/IFAS NFREC Beef Unit suffered massive damage from Hurricane Michael in October of 2018. Data presented here represent the breeding season that happened only five months after Hurricane Michael.

Synchronization protocols (Figure 2, panels A and B) are a precise sequence of hormonal treatments that are administered to a group of females in order to synchronize heats. Drugs used were GnRH analogs, prostaglandin F2-alpha analogs (PGF), and progesterone from intravaginal releasing devices, such as a CIDR. All females submitted to the protocol were artificially inseminated; pregnancy rates from that insemination varied from $28 \%-56 \%$, depending on the female category (Figure 2, panels C and D, blue bars). After AI, females were exposed to clean-up bulls. The final pregnancy rates of the breeding season, including pregnancies both from the AI and from natural service, are shown in Figure 2, panels $\mathrm{C}$ and $\mathrm{D}$ (orange bars). There were fetal losses that occurred between the last pregnancy check and the expected calving date. These losses were usually low and varied from $0 \%-7 \%$, depending on the female category (Figure 2, panels E and F, blue bars). Finally, calf losses occurred between calving and weaning and averaged 3\%-6\% (Figure 2, panels E and F, orange bars).

\section{Summary}

In this article, we reported local reproductive performance information, using breeds that are relevant to the production systems in the state. We confirmed that it is possible to obtain adequate pregnancy rates to A.I. + natural service within a limited breeding season. We show that reproductive success and fetal and calf survival varied according to the female category.

\section{Acknowledgments}

We thank the Gainesville and the Marianna Beef Units' staff for their hard work, as well as the students who collaborated on data collection and compilation. We are grateful to Zoetis for the donation of synchronization drugs (Gainesville and Marianna) and Select Sires for the donation of semen (Marianna). 
Table 1. Overview of the reproductive management of the UF Beef Units.

\begin{tabular}{|l|c|c|}
\hline \multicolumn{1}{|c|}{ Variable } & Gainesville & Marianna \\
\hline Main breed composition & Angus x Brahman crossbreds & Angus, Brangus, Braford \\
\hline Date breeding season started: heifers & $2 / 8 / 2019$ & $3 / 22 / 2019$ \\
\hline Date breeding season started: cows & $2 / 15 / 2019$ & $3 / 29 / 2019$ \\
\hline Duration of breeding season & $85-100$ days & $80-85$ days \\
\hline Days after Al bulls were introduced & 15 & $4-8$ \\
\hline Bull:Cow ratio & $1: 40$ & $1: 22$ \\
\hline
\end{tabular}

